\title{
The event of ground level enhancement of solar cosmic rays on October28, 2003: the spectrum in a wide energy range
}

\author{
G.F. Krymsky, V.G. Grigoryev, S.A. Starodubtsev, S.N. Taneev, P.Yu. Gololobov ${ }^{\mathbf{1}}$ \\ Yu.G. Shafer Institute of Cosmophysical Research and Aeronomy of SB RAS \\ 31 Lenin ave., 677980 Yakutsk, Russia \\ E-mail:krymsky@ikfia.sbras.ru, grig@ikfia.sbras.ru, starodub@ikfia.sbras.ru, \\ taneev@ikfia.sbras.ru, gpeter@ikfia.sbras.ru
}

\begin{abstract}
We present the results of spectrum analysis of the event of ground level enhancement of solar cosmic rays on October 28, 2003 (GLE65) in the widest range of energies. The energy spectrum of cosmic rays is studied on the basis of direct measurements of solar particle fluxes aboard the ACE, GOES and WIND spacecraft, as well as by data recorded by the worldwide neutron monitor network. In the relativistic energy range the estimations of spectrum have been obtained using the effective energy method proposed by the authors. It has been established that in this event the energy spectrum of solar cosmic rays extends from $\sim 40 \mathrm{keV}$ to $\sim 5 \mathrm{GeV}$ and it is described by a power function with an exponential cut-off in the field of relativistic energies. To find out the nature of solar cosmic rays the quasi-linear theory of regular acceleration (diffusive shock acceleration) of charged particles by shock waves in the low solar corona developed at ShiCRA of SB RAS is used. It has been concluded that the process of acceleration of solar cosmic rays was over at a distance of 4 solar radii.
\end{abstract}

The 34th International Cosmic Ray Conference

30 July- 6 August, 2015

The Hague, The Netherlands

${ }^{1}$ Speaker 


\section{Introduction}

One of the key problems of the Sun's physics is to find out mechanisms of generation of solar cosmic rays (SCRs). This raises a number of important questions, for example: 1 . What energies the particle acceleration is possible up to? 2. Where and under what processes does it happen and what is the form of the energy spectrum?

On the basis of the analysis of experimental data on energetic particles fluxes, at present time it is common practice to divide the events of SCR flares (or solar energetic particles (SEP) events) into following two classes: impulse and gradual ones (see, for example, [1]). Such division is caused by a distinction of properties of the observable events (the chemical compound of devices, time of course of an event in X-rays and profiles of registered particles, type of bursts of a radio emission etc.). It is customary to assume that two classes of events are caused by different mechanisms of particle generation on the Sun - in magnetic reconnecting in the Sun's lower corona and on the coronal shocks, respectively [2]. The SEP events, which are accompanied by a generation of relativistic particles, are observed rather seldom and the collected statistics is insufficient for getting unambiguous conclusions about the conditions of their formation and generation mechanisms. Investigations of such events continue to remain actual. The necessity of study of the energetic spectrum in a wide range creates the known methodical difficulties connected to the use of neutron monitor data.

In this work the ground increase of SCRs on October 28, 2003 is studied. For the events of SCR flares which are registered by the difference detectors on the Earth the term «Ground Level Enhancement» with an assignation of corresponding number has been accepted. In correspondence with it, the event considered by us is known in the scientific literature as GLE65. The powerful solar flare of the X17/4B amount which has happened at 11:10 UT on October, 28th 2003, in the active field of RA 10486 with the coordinates S16E08 (http://umbra.nascom.nasa.gov/SEP/seps.html) has served as a reason of it. The flare has created an ejection of coronal mass of the solar substance which is accompanied by a strong shock wave [3], therefore, there are causes to refer it to the class of gradual events. Analyzing this event, the method of effective energies suggested by us earlier is applied [4]. In determining the energetic spectrum of SCRs the special attention is paid to the estimation of methodical errors in the determination of particle energy. The reliability of the obtained results depends on it. The spectrum of increase of SCRs is determined during the isotropic phase of event. Further for clearing up the nature the obtained spectrum is compared with the results of quasi-linear theory developed at ShICRA of Siberian Branch of the Russian Academy of Science recently [5].

\section{Determination of energy spectrum of SCRs by measurements of neutron monitors using a method of effective energies}

Unfortunately, at present time it is impossible to carry out measurements of SCR fluxes in the whole measured energy range with the help of uniform device. The use of data of direct measurements of solar particle fluxes aboard space vehicles allows to obtain the experimental SCR spectra only in the non-relativistic energy range. At the same time the spectra of relativistic SCRs generated during the powerful solar events are determined by indirect measurements of SCR intensity with the ground detectors, mainly, neutron monitors. The use of data collection of 
coincident observations on the Earth and in space environment allows to obtain energy SCR spectra in the widest energy range from tens $\mathrm{keV}$ to tens $\mathrm{GeV}$, and further to make the informative conclusions about generation mechanisms on the Sun.

In using the data of ground measurements the essential problems with the determination of energy and SCR fluxes and errors of their measurements appear. It is connected with two following circumstances: 1 . Ground devices measure not SCR flux but they register the rate of the account of secondary particles. Mainly, these are neutrons and muons born in interacting of cosmic rays with an Earth's atmosphere; 2. Ground devices register particles in the integral energy range beginning from some minimum station depending on a geographical location of the station where they are installed. In this case, the minimum energy of particles registered at polar stations, is determined by the atmosphere's thickness only and accounts for about 433 $\mathrm{MeV}$. It corresponds to particles with the rigidity $R=1 \mathrm{GV}$ whereas the nominal rigidity of geomagnetic cut-off in polar regions is obviously less than $1 \mathrm{GV}$. Unlike it, at mid- and low latitudinal stations the minimum energy of registered particles is determined by the value of geomagnetic cut-off $R_{C}>1 \mathrm{GeV}$. To make recalculation from the registered intensity of atmospheric neutrons to the proton fluxes beyond the bounds of the Earth's magnetosphere it is necessary to consider these circumstances correctly. For this purpose it is necessary to know the receiving characteristics of stations i.e. the geographical coordinates, height above sea level, receiving cones and coupling coefficients of devices which vary with a cycle of solar activity [6]. Therefore, to determine the energy and SCR fluxes using the data of neutron monitors, we use here the method of effective energies suggested by us earlier [4].

The essence of this method consists in the determination of some effective impulse $p_{e f}$ (or energy $E_{e f}$ ) for each neutron monitor which has registered a SCR event, at which the calculated absolute SCR flux $(J)$ does not noticeably change when the index of energy distribution $r$ changes a little. The contribution of particles of various impulses $p$ into the increase of SCR intensity observed with the neutron monitor is proportional to the multiplicity of generation of secondary particles in the Earth's atmosphere $m(p)$ and spectrum of solar particles $J_{s}(p)$. The multiplicity determines a sensitivity of neutron monitor to primary cosmic rays and undergoes the cut-off from the side of low energies, and the spectrum promptly decreases with the energy growth. The product of these functions represents a curve with a sharp maximum decreasing promptly on either side from it. This circumstance is used by us in the suggested method [4].

\section{Data of observations}

To determine the energy spectrum of SCRs in the widest energy range we have used the data of registration of particles in the Earth-orbital space and on the Earth. To obtain the energy spectrum of SEPs in the non-relativistic energy spectrum we have used the corrected 5-min registration data of charged particles aboard three following spacecrafts: ACE located near to the point of libration L1, GEOS-10 being on a geostationary orbit, and WIND which was at the magnetosphere's tail at that time at a distance of about 150 radii of the Earth, and whose influence on the energetic particles is minimum. It has allowed us to analyze the data of measurements in the following 17 differential energy channels: 1 . Eight channels of registration 
of ions (mainly protons) of the EPAM/LEMS120 device installed aboard the ACE spacecraft : 0.047-0.66, 0.066-0.114, 0.114-0.190, 0.190-0.310, 0.310-0.580, 0.580-1.050, 1.050-1.890 and 1.890-4.750 MeV; 2. Seven channels of registration of protons with the TELESCOPE and DOME detectors installed aboard the GOES-10 spacecraft in the energy range : 0.8-4, 4-9, 9-15, 15-40, 40-80, 80-165 and 165-500 MeV; 3. Two channels of measurements of protons in the energy range 19-28 and 28-72 MeV of the EPACT device installed aboard the WIND spacecraft. Data of measurements with these spacecraft are in open access: http://www.srl.caltech.edu/ACE, http://spidr.ngdc.noaa.gov and http://cdaweb.gsfc.nasa. gov/daweb/sp phys. To analyze the SCR in the range of relativistic energies we have used the 1- and 5-min data of measurements at 16 world neutron monitor network stations with different thresholds of the geomagnetic cut-off on $R_{C}$ which the increase of solar particles has been registered. These are Mc Murdo ( $R_{C}=0.01$ GV), Terre Adelie (0.01), Thule (0.10), Inuvik (0.18), Nain (0.40), Apatity (0.65), Oulu (0.81), Sanae (1.06), Kerguelen (1.19), Yakutsk (1.65), New Arc (1.97), Magadan (2.10), Kiel (2.29), Moscow (2.46), Larc (3.00) and Irkutsk (3.66).

\section{Energy spectrum of SCRs in the event of GLE65}

The joint analysis of measurement data mentioned above has allowed us to determine the energetic spectrum of SCRs in the widest energy range from tens $\mathrm{keV}$ up to several $\mathrm{GeV}$ which is observed on the Earth's orbit . Fig. 1 shows the SEP event registered with these spacecraft and presented in the form of dependence on the time of observable SEP flux.

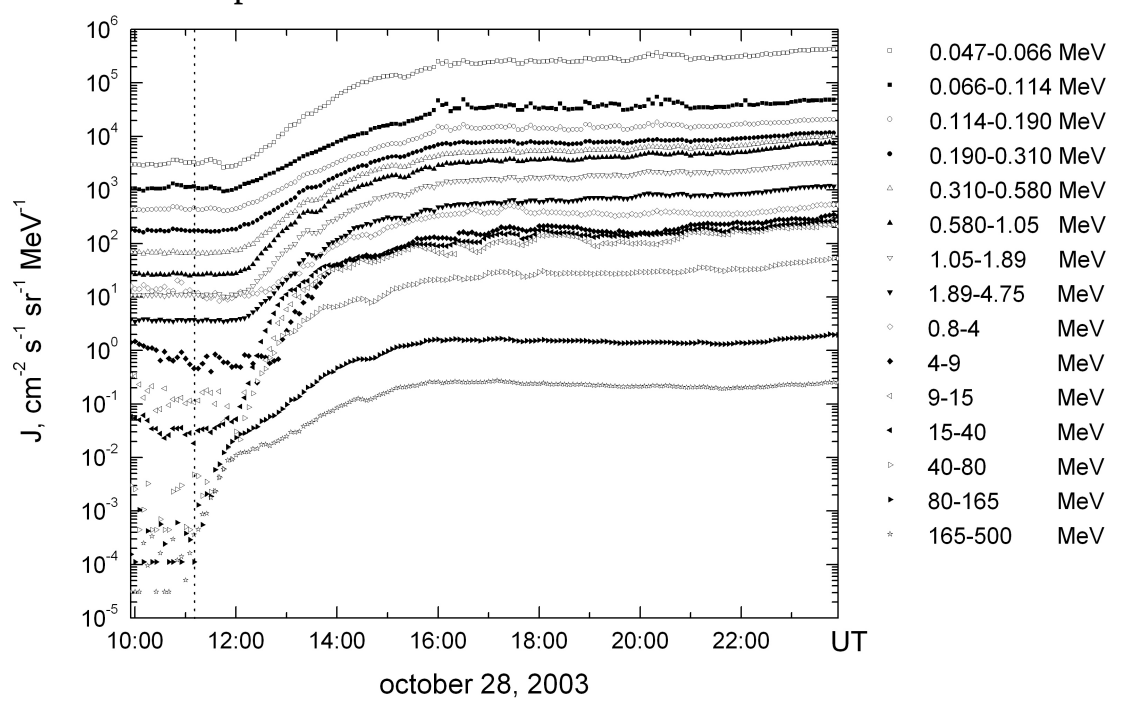

Figure 1: The SEP event on October 28, 2003 by 5-min data of direct measurements of proton fluxes in 15 differential energy channels aboard spacecraft of ACE (from 0.047 to $4.75 \mathrm{MeV}$ ) and GOES-10 (from 0.8 to $500 \mathrm{MeV}$ ). The vertical dashed line marks the time of burst on the Sun. Measurements of particle fluxes in the corresponding differential energy channels are marked by symbols. The legend is on the right.

As an example of measurements made on the Earth, the dependence on time of the cosmic ray intensity registered by neutron monitors at the Mc Murdo, Terre Adelie, Yakutsk and Irkutsk stations is given (Fig. 2). A peculiarity of this event was the fact that very prompt (less than 10 mines) arrival of SCRs from the flare moment on the Sun in the Earth's orbit and the increase of 
solar particle flux was manifested in the very low energies (Fig. 1 and 2). From Fig. 2 it is seen that the value of ground increase for different stations was changing from a few per cent up to approximately 50 ones, in this case for the initial phase of event the considerable anisotropy (Fig. 2a and b) is typical. For the determination of GLE spectrum we have chosen the moment of time of 14:00 UT when the cosmic ray intensity became adequately isotropic (Fig. 2a-d).
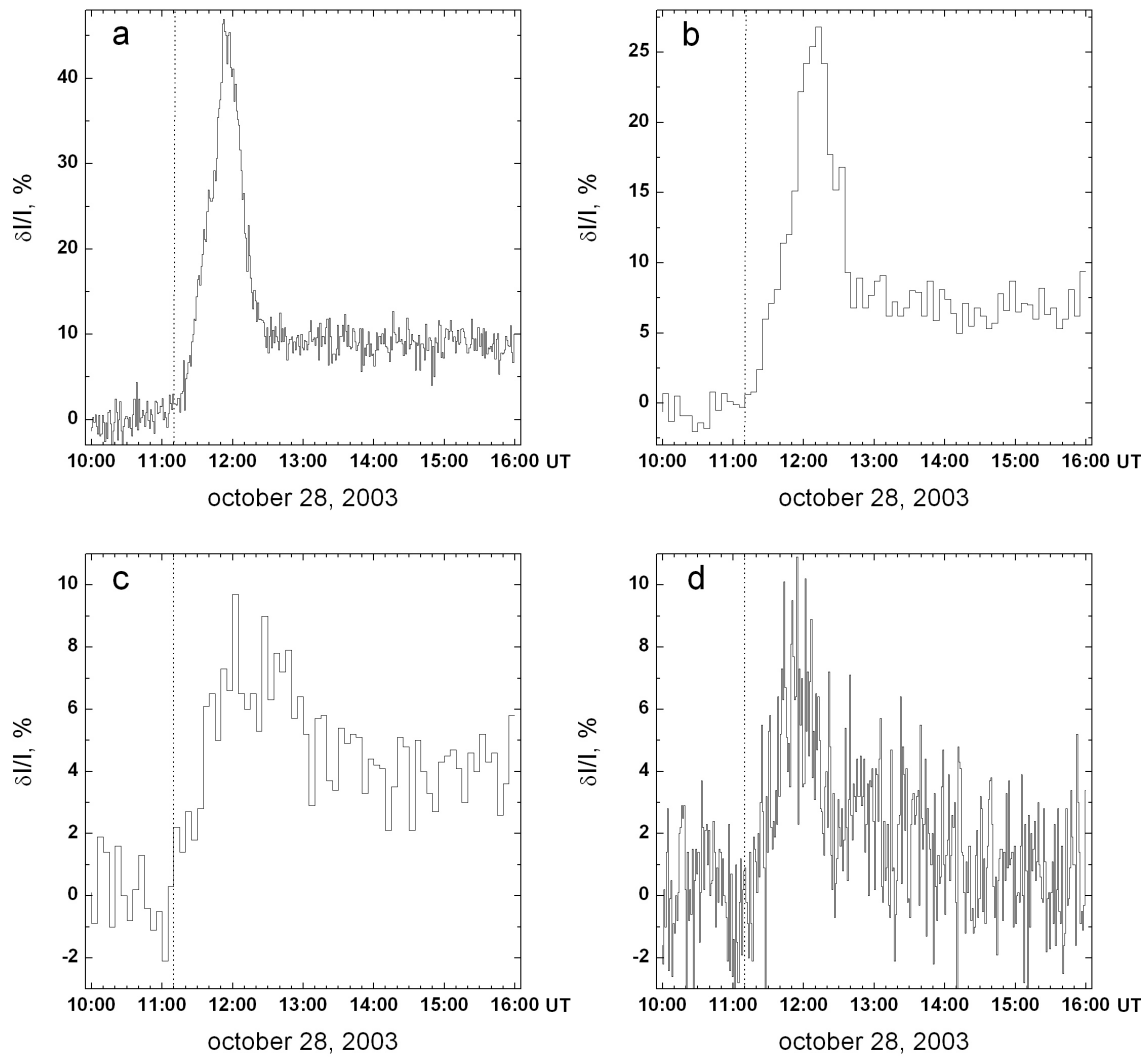

Figure 2: The GLE65 event on October 28, 2003 by measurements of neutron monitors of the Mc Murdo (1-min data, the panel a), Terre Adelie (5-min data, the panel b), Yakutsk (5-min data, the panel c) and Irkutsk (1-min data, the panel d) cosmic ray stations. The vertical dashed line marks the time of flare on the Sun.

Fig. 3 presents the SCR energy spectrum in the energy range from tens keV up to several $\mathrm{GeV}$ obtained by diffusive shock acceleration. As is seen from it, the whole observable spectrum of SCRs can be, on the whole, (except the energy range from 4 to $80 \mathrm{MeV}$ ) is described by a power function with an exponential cutt-off of the form: $J(E)=J_{0} E^{-\Upsilon} \exp \left(-E / E_{0}\right)$. It is in agreement with results obtained earlier by various authors in studying other GLE events [7, 8].Taking into account this circumstance, the SCR spectrum obtained by us in the studied event can be approximated by a function $J(E)=350 E^{-1.4} \exp (-E / 450 \mathrm{MeV})$ with a satisfactory accuracy (curve 1). Let's note that the uncertainties of measurements of SCR flux values aboard spacecraft are small, they are less than values of SCR fluxes, and errors of determination of effective energies of particles by data of ground measurements in the considered case do not exceed $20 \mathrm{MeV}$, so these uncertainties are not seen in Fig. 3. 


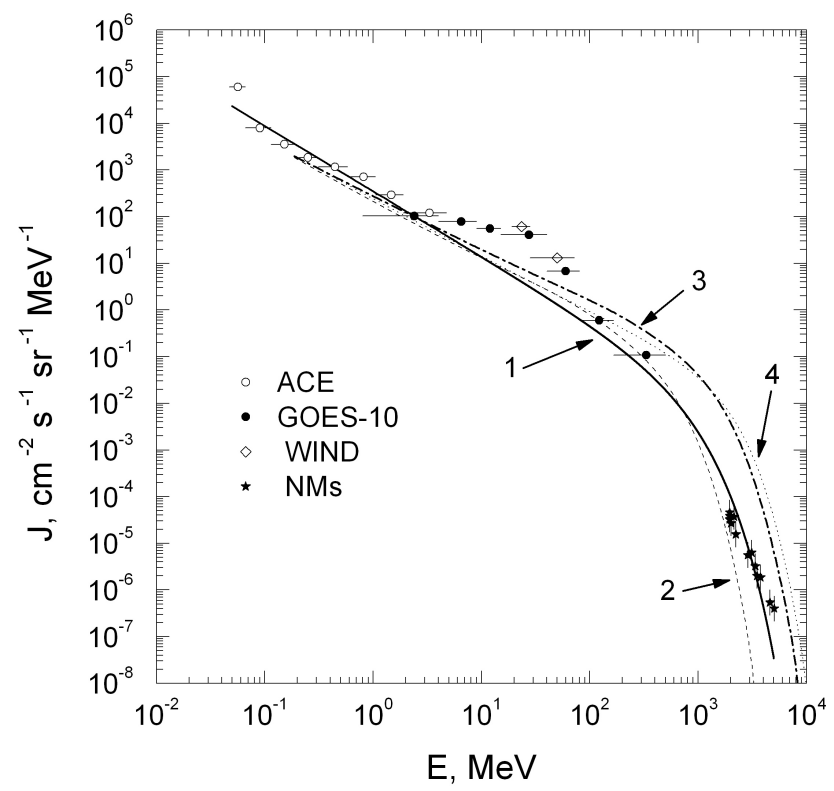

Figure 3: The energy spectrum of SCRs at 14:00 UT on October 28, 2003 in the widest range of energies by measurements aboard the ACE, GOES-10 and WIND spacecrafts, and also using the neutron monitor network. The widths of differential energy channels are shown by horizontal bars, the uncertainties of measurements of SCR fluxes are shown by vertical bars. The curves show the following: 1 - the approximation by function $J(E)=350 E^{-1.4} \exp (-E / 450 \mathrm{MeV})$; 2, 3 and 4 - calculations by the model [5].

\section{Comparison with the results of application of the quasi-linear theory of acceleration of SCRs on coronal shocks}

In many cases the chemical compound, charge state [9], and also the dependence of SCR intensity on the time registered by the ground detectors [10], testify that in case of gradual (unlike impulse ones) solar events the generation of SCRs occurs in front of shock waves generated by a supersonic movement of substance mass ejected from the Sun's surface. The theoretical analysis shows that physical parameters in the Sun's crown ensure the conditions of realization of effective process of the regular acceleration of ions which is capable to form the SCR spectrum with the required properties $[8,11,12]$. The investigations of process of SCR acceleration made up to present time are of approximate, estimating character $[8,11,13,14,15$, 16]. All of them are based either on a simplified linear flat-wave theory variant, or do not take into account a real distribution of coronal plasma parameters.

The work [12] has shown an important value of specified factors in generating of particles by means of the regular mechanism of acceleration in fronts of shock waves [17]. In particular, in the work [12] it is shown that the consistent theory of regular SCRs acceleration should include the following: the account of real geometry of shock wave and dependences of its parameters on the time for the whole duration of its distribution from the upper crown of the Sun to the Earth's orbit; the account of turbulence generation by means of the accelerated particles which in its turn, defines the character of their diffusion; the account of inverse action of pressure of the accelerated particles on a structure and dynamics of shock wave. The developed linear theory of SCR acceleration [12] is the first example of application of the 
theory of regular acceleration of charged particles with the account of extremity of sizes of a shock wave (in a spherical approximation); the account of adiabatic deceleration of the accelerated particles, and also the consideration of real parameters of the solar crown plasma for explanation of the phenomenon of SCR generation. Further, the account of self-consistent generation of Alfven waves by accelerated particles has led to creation of the quasi-linear theory of regular acceleration of SCRs up to relativistic energies in front of coronal shock wave [5]. For physical explanation of a certain spectrum of studied event we have drawn this theory. On its base we have made three calculations. In correspondence with this work [12], we have accepted that the shock wave speed $V_{S}$ in the solar corona is connected with the ejection velocity of coronal mass of the solar substance $V_{C M E}$ by a following relation: $V_{S}=V_{C M E} \sigma /(\sigma-1)$, in this case the degree of compression in front of the strong shock wave $\delta=4$, and the observed ejection velocity $V_{C M E}=2500 \mathrm{~km} / \mathrm{s}$ [3]. Thus, the shock wave velocity in the solar corona has been taken as $V_{S}=3300 \mathrm{~km} / \mathrm{s}$, and the rate of particle injection in the acceleration process $\zeta=10^{-5}$. Plasma parameters in the solar corona have been accepted the same, as well as in [5]. In the first variant of calculation the magnetic field intensity in the base of solar corona has been taken as $B=3$ Gs. In the second one is $B=2$ Gs. The third variant of calculation differs from the first one by a dependence of spectrum of the background Alfven turbulence $E_{W}$ on the frequency $v\left(E_{W}(v) \sim v^{-\lambda}\right.$, where $\lambda$ is an index). More detailed description of a selection of background Alfven turbulence can be found in [5]. In the third variant of calculation it has been accepted $\lambda=3 / 2$, instead of the value $5 / 3$ used usually. The calculations carried out show that the process of SCR acceleration in this event was over in propagating of a shock wave up to height about $4 R_{\odot}$, where $R_{\odot}$ is the Sun's radius. This value depend on several factors defining the efficiency of particle acceleration on shock waves in the solar corona which are considered in details in [5, 12], respectively. Further, for the comparison of spectra of the particles accelerated on the Sun with the observable spectra, we have used the simplified model of purely diffusion distribution of SCRs up to Earth's orbit [12].

From Fig. 3 it is seen that theoretically calculated spectra (curves 2, 3 and 4) mainly reproduce the observational measurements on Earth's orbit correctly and show a possibility to determine the most suitable spectrum using the choice of several parameters.

On closer consideration of Fig. 3 it is seen that in the energy range of $\sim 10 \mathrm{MeV}$ a bump is observed in the spectrum. The nature of similar bumps which are often observed in the particle spectra in their accelerating in fronts of shock waves under various astrophysical conditions, was repeatedly considered in $[5,12,18,19]$, however, there is no still the complete clearness in this problem.

The analysis of the obtained results allows to conclude that the theory developed in [5], allows to explain the mechanism of SCRs generation in the event of GLE65 on the basis of regular acceleration of particles by shock waves in the lower solar corona.

\section{Conclusion}

Thus, on the basis of investigations carried out, one can make the following conclusions: 1. In the method of estimation of the differential energy SCR spectra suggested by us earlier by 
data of ground measurements [4] the methodical errors of determination of effective energy of solar particles are rather insignificant and account for less than $20 \mathrm{MeV}$; 2. For the event of GLE65 the SCR spectrum observed in a wide energy range from tens $\mathrm{keV}$ up to $\sim 10 \mathrm{GeV}$ can be presented in the form of power function with an exponential cut-off of the following view: $J(E)=350 E^{-1.4} \exp (-E / 450 \mathrm{MeV})$ that finds its natural explanation within the framework of quasi-linear theory of the regular acceleration of particles in the lower solar corona [5]; 3. In the considered event of GLE65 the process of acceleration of SCRs in front of the coronal shock wave was over at height of up to $4 R_{\odot}$, i.e. below field of the current of solar wind from the Sun's surface.

\section{Acknowledgments}

The authors thank Goddard Space Flight Center (GSFC), National Oceanic and Atmospheric Administration (NOAA) and ACE Science Center for the data of measurements aboard spacecraft, and also the management of the Oulu cosmic ray station (Finland) for the data of measurements of the world neutron monitor network for the events of GLE at the website (http://gle.oulu.fi).This work was supported by the Russian Foundation for Basic Research, grant No.13-02-00941, by Program No.31 of the Presidium of the Russian Academy of Sciences, and by RF Presidential Grant in Support of Leading Scientific Schools NS3269.2014.2.

\section{References}

[1] D. V. Reames, Rev.Geophys., 33, 585 (1995).

[2] D. V. Reames, Space Sci. Rev., 175, 53 (2013).

[3] W. B. Manchester IV, A. Vourlidas, G. T oth, N. Lugaz, I. I. Roussev, I. V. Sokolov, T. I. Gombosi, D. L. De Zeeuw, M. Opher), Astrophys. J., 684, 1448 (2008).

[4] G. F. Krymsky, V. G. Grigoryev, S. A. Starodubtsev, JETP Lett., 88, 411 (2008)

[5] E. G. Berezhko, S. N. Taneev, Astron. Lett., 39, 393 (2013)

[6] L. I. Dorman, Cosmic rays in the Earth's atmosphere and underground (Kluwer Academic Publishers, The Netherlands, 2004)

[7] J. L. Lovell, M. L. Duldig, J. E. Humble, J. Geophys. Res.: Space Phys., 103, 23733 (1998)

[8] D. C. Ellison, R. Ramaty, Astrophys. J., 298, 400 (1985).

[9] D. V. Reames, AIP Conf. Proc., 374, 35 (1996).

[10] S. W. Kahler, Astrophys. J., 428, 837 (1985).

[11] M. A. Lee, J. M. Ryan, Astrophys. J., 303, 829 (1986).

[12] E. G. Berezhko, S. N. Taneev, Astron. Lett., 29, 530 (2003)

[13] G.P. Zank, W. K. M. Rice, C. C. Wu, J. Geophys. Res., 105, 25079 (2000).

[14] W. K. V. Rice, G.P. Zank, G. Li), J. Geophys. Res., 108, 1369 (2003).

[15] C. K. Ng, D. V. Reames, A. J. Tylka, Astrophys. J., 591, 461 (2003).

[16] L. I. Miroshnichenko, J. Perez-Peraza, Int. J. Modern Phys. A, 23, 1 (2008)

[17] G. F. Krymskii, Sov. Phys. Dokl., 22, 327 (1977).

[18] E. G. Berezhko, G. F. Krymskii, Sov. Phys. Usp., 31, 27 (1988).

[19] E. G. Berezhko, S. N. Taneev, S. I. Petukhov, A. A. Turpanov, Astron. Lett., 22, 260 (1996) 